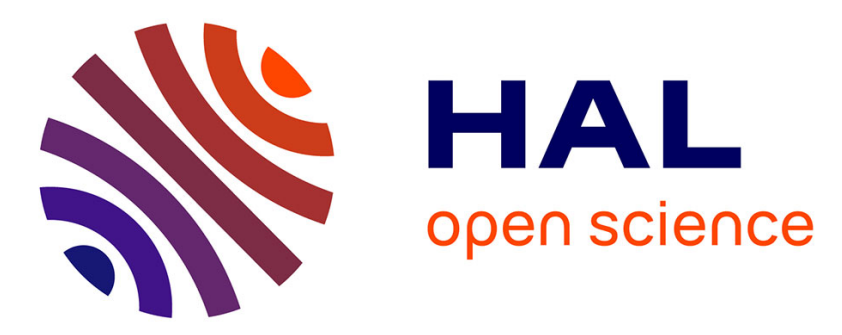

\title{
Numeric and symbolic knowledge representation of cerebral cortex anatomy: methods and preliminary results.
}

Olivier Dameron, Bernard Gibaud, Xavier Morandi

\section{To cite this version:}

Olivier Dameron, Bernard Gibaud, Xavier Morandi. Numeric and symbolic knowledge representation of cerebral cortex anatomy: methods and preliminary results.. Surgical and Radiologic Anatomy, 2004, 26 (3), pp.191-7. 10.1007/s00276-003-0204-0 . inserm-00152193

\section{HAL Id: inserm-00152193 https://www.hal.inserm.fr/inserm-00152193}

Submitted on 29 Jun 2010

HAL is a multi-disciplinary open access archive for the deposit and dissemination of scientific research documents, whether they are published or not. The documents may come from teaching and research institutions in France or abroad, or from public or private research centers.
L'archive ouverte pluridisciplinaire HAL, est destinée au dépôt et à la diffusion de documents scientifiques de niveau recherche, publiés ou non, émanant des établissements d'enseignement et de recherche français ou étrangers, des laboratoires publics ou privés. 


\section{Numeric and symbolic knowledge representation of cerebral cortex anatomy: methods and preliminary results}

Received: 14 November 2002/ Accepted: 15 September 2003/Published online: 30 April 2004

(C) Springer-Verlag 2004

\begin{abstract}
The human cerebral cortex anatomy describes the brain organization at the scale of gyri and sulci. It is used as landmarks for neurosurgery as well as localization support for functional data analysis or inter-subject data comparison. Existing models of the cortex anatomy either rely on image labeling but fail to represent variability and structural properties or rely on a conceptual model but miss the inner 3D nature and relations of anatomical structures. This study was therefore conducted to propose a model of sulco-gyral anatomy for the healthy human brain. We hypothesized that both numeric knowledge (i.e., image-based) and symbolic knowledge (i.e., concept-based) have to be represented and coordinated. In addition, the representation of this knowledge should be application-independent in order to be usable in various contexts. Therefore, we devised a symbolic model describing specialization, composition and spatial organization of cortical anatomical structures. We also collected numeric knowledge such as 3D models of shape and shape variation about cortical anatomical structures. For each numeric piece of knowledge, a companion file describes the concept it refers to and the nature of the relationship. Demonstration software performs a mapping between the numeric and the symbolic aspects for browsing the knowledge base.
\end{abstract}

O. Dameron · B. Gibaud · X. Morandi $(\bowtie)$

Laboratoire IDM-UPRES-EA 3192,

Faculté de Médecine de Rennes,

Avenue du Professeur Léon Bernard,

35043 Rennes cedex, France

X. Morandi

Laboratoire d'Anatomie et d'Organogenèse,

Faculté de Médecine de Rennes,

Avenue du Professeur Léon Bernard,

35043 Rennes cedex, France

E-mail: xavier.morandi@chu-rennes.fr

Tel.: + 33-2-99284277

Fax: + 33-2-99284180
Keywords Brain atlas - Neuroanatomy - Ontology

Sulcus · Visualization $\cdot$ Computer-aided surgery

\section{Introduction}

Modern whole-brain imaging techniques have allowed the neuroscience community to gather a detailed inventory of information on the cortical anatomy of individual brains. Information used to be presented within paper atlases [10, 30, 37, 38]. More recently, the emergence of computerized atlases enhancing both the navigation in the atlas and the representation of the three-dimensional (3D) nature of brain anatomy has helped to launch a new era of human brain representation $[1,17,20,29]$. This approach is based on the segmentation of the anatomical structures in images, eventually completed with textual or hyper-textual descriptions, and constitutes the "image-based approach" of anatomical knowledge representation. The usefulness of these atlas systems in clinical decision support depends on how closely the brain of individual subjects matches the representation of anatomy in the atlas. Indeed, this method does not take into account the great inter-individual variability of the sulco-gyral anatomy [12, 36, 39, 41]. Moreover, a part of the knowledge is represented in an implicit way, i.e., within the images. These two drawbacks limit the possibilities of reuse of these atlases by software in applications such as image-guided neurosurgery, research or teaching in the neurosciences $[18,19,21,26]$.

This need for an explicit and more formalized knowledge set about cerebral cortex anatomy triggered the creation of symbolic models [27, 35]. Current terminology systems such as NeuroNames [5] in the field of neuroanatomy provide limited semantic information for a set of concepts. Yet, they represent neither properties nor relations for these concepts, except for the is-part-of relation, but this often leads to tangled hierarchies [6, 35]. The UMLS (Unified Medical Language System) [3] 
relies on a semantic network to map biomedical vocabularies from various sources. The integration of NeuroNames into the UMLS semantic network in 1992 was a step to address this issue by adding some of the missing relationships. Yet further specifications such as the definition of identity criteria for the concepts are required. The two leading projects regarding the symbolic representation of anatomy are Digital Anatomist $[6,35]$ and GALEN (Generalized Architecture for Languages, Encyclopedias and Nomenclatures in medicine) [33]. They are representatives of a "concept-based approach". However, they focus on organs and none of them offers an accurate description of the sulco-gyral anatomy organization and variability. Moreover, the concept-based approach cannot accurately account for the shape of anatomical structures.

We hypothesized that anatomical knowledge encompasses both numeric elements, i.e., description primitives extracted from anatomical images of actual brains, and symbolic ones, i.e., description primitives based on language and conveying meaning. Therefore, the image-based approach and the concept-based approach are complementary. The former addresses 3D shape representation using both magnetic resonance imaging (MRI) and computer graphics. The latter provides an abstract structural description. Our goal was:

(1) to gather numeric knowledge, particularly in three dimensions,

(2) to propose a consistent symbolic model suitable for representing the concepts of sulco-gyral anatomy of the brain cortex,

(3) to allow mapping between these two models.

The achievement of these three aims relies on web technologies in order to minimize usage constraints. Our preliminary results focus on the concept of a symbolic model adapted to brain cortex anatomy specificities and on the coordination of the numeric and symbolic aspects.

\section{Materials and methods}

Anatomical imaging and segmentation step

Anatomical data were obtained from 2D and 3D MR images of the brain. For this preliminary study, a database was created by computing the average of nine examinations of a single subject. These images were averaged and aligned by ANIMAL software [9].

A T1-weighted 3D spoiled gradient MRI examination was performed using a 1.5 T MR scanner (General Electric Medical Systems, Milwaukee, Wis.). Acquisition parameters were as follow: TR $33 \mathrm{~ms}$, TE $3 \mathrm{~ms}$ and one excitation; flip angle $45^{\circ}$; 124 contiguous sagittal $1.3 \mathrm{~mm}$ thick slices; 256×256 matrix; and $240 \mathrm{~mm}$ FOV. An isotropic volume was generated from the T1-weighted MRI anatomical data set. To obtain the segmentation of the cortical sulci from the anatomical MRI volume, we performed automatic brain segmentation and classification of white matter, gray matter and cerebrospinal fluid [2]. The external traces of the sulci at the cortical surface were then automatically extracted [23]. According to a method developed in our laboratory, cortical folds were extracted using active contours and curvature analysis [22]. The median surface of relevant sulci was generated from their external traces. This method allowed an automatic $3 \mathrm{D}$ representation of the cortical sulci. A 3D scene composed of all the external traces and of the medial surfaces of the selected sulci was then automatically generated.

\section{Anatomical model}

Our work addressed on one hand the modeling and on the other hand the representation of both numeric and symbolic anatomical knowledge.

The symbolic anatomical knowledge structure was composed of different classes of concepts, of their properties and of the relationships that associate them (Fig. 1). Thus, for example, we have modeled the various possible parts of a gyrus, or the fact that the central sulcus is a kind of sulcus. This structure was modeled with a UML (Unified Modeling Language, a widespread object-oriented modeling language) [4] class diagram, and completed by textual definitions in order to convey the intended meaning.

The symbolic anatomical knowledge was represented in an XML file [16] (eXtensible Markup Language, language allowing representation of information by explicitly stating its structure. For example, $<$ Designation terminology $=$ 'NeuroNames' $>$ Central Sulcus $</$ Designation $>$ indicates that the string "Central Sulcus" is a designation of an anatomical concept in the NeuroNames terminology). The grammar of an XML document can be defined in a DTD (Document Type Definition; for example, it can state that every anatomical concept has at least one designation) [16]. This DTD was manually generated from the previous UML class diagram. The XML file contains the list of the concepts and relationships. Each one has a unique identifier. For every concept, references to well-established terminologies such as NeuroNames [5] or Nomina Anatomica [28] were provided whenever possible.

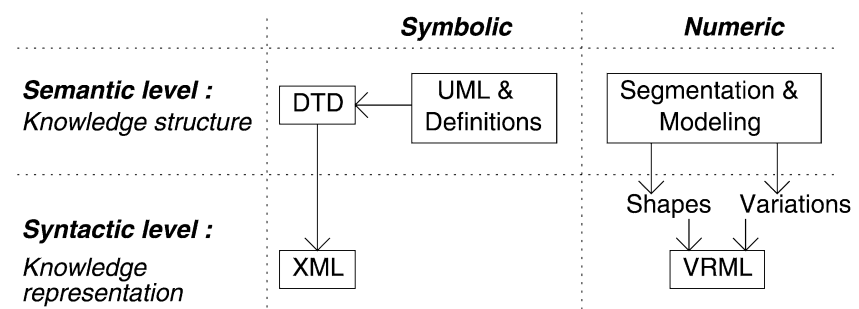

Fig. 1 Modeling and representation steps of anatomical knowledge 
The segmentation step provided some prototypical shape models for the cortical structures.

The representation of numeric anatomical knowledge consisted in generating 3D scenes as VRML files (Virtual Reality Modeling Language) [7]. The various numeric structures that were represented were regions of the $3 \mathrm{D}$ scene.

The links between the symbolic and the numeric elements were stored in companion XML files. Every link had a role describing the nature of the relationship between the resources. These links permitted the labeling of the numeric elements against the symbolic ones. They have been manually established by an expert. We devised an application in Java (object-oriented programming language) highlighting the mapping between these two aspects.

\section{Results}

The results of this study were the creation of (1) a formalized model as a UML class diagram for cortex anatomy concept classes, completed by textual definitions to convey the semantics, (2) an anatomical knowledge base, instantiated from the former model, and (3) a mapping between the numeric and symbolic aspects. An application demonstrates the browsing of the knowledge base and of the 3D scenes.

\section{Cortex anatomy model}

The cortex anatomy model described the various classes of concepts of cerebral cortex anatomy, and their organization. As classes of concepts, we identified Sulcus, Gyrus, Pars of a Gyrus, Operculum, Pli De Passage,Lobe and Hemisphere. As for relations, we distinguished specialization, partitionand connection relations.

Specialization relation was typically the is-a-kind-of relation. The properties of the most general concept also held for the more specific. For example, the central sulcus is-a-kind-ofSulcus. As a Sulcus is filled with cerebrospinal fluid (CSF), the central sulcus is also filled with CSF.

Partition relations held between a compound and its components. They were the is-direct-anatomical-partof,is-anatomical-part-of and the is-segment-of relations. The is-direct-anatomical-part-of relation held between a Lobe and a Hemisphere, a Pars of Gyrus and a Gyrus, or between a Gyrus, an Operculum or a Pli De Passage and a Lobe. Every structure except Hemisphere was the direct anatomical part of exactly one other structure. The is-anatomical-part-of relation was the transitive closure of is-direct-anatomical-part-of, so that a cortical structure could be an anatomical part of many other cortical structures. For example, the precentral gyrus is-direct-anatomical-part-of the frontal lobe and is-anatomical-part-of the hemisphere. The is-segment-of relation described that a Sulcus was a part of another Sulcus. The properties of the compound did not necessarily hold for its components, and conversely. For example, the frontal lobe was contiguous with the parietal lobe, but the superior frontal gyrus, which is-anatomical-part-of the frontal lobe, was not contiguous with the parietal lobe.

The connection relation was used to provide a topological description of the anatomical structures. It was essential for identifying these structures. We used the binary is-continuous-to relation to represent the adjacency with no interruption of two structures among Pars, Gyrus, Operculum, Pli De Passage, Lobe or Hemisphere. For instance, such a relation held between the precentral gyrus and the inferior frontal gyrus. We used the ternary is-separated-from-...-by- relation to represent the separation of two structures among Pars, Gyrus, Operculum, Pli De Passage or Lobe by a Sulcus. For instance, this relation represents the delimitation of the precentral gyrus and the postcentral gyrus by the central sulcus.

In addition, contrary to the specialization relations, the composition and the topological relations are affected by intra- and inter-individual variability. We represented this variability by complementing the relationships with a probability-of-existence property.

\section{Knowledge base}

The knowledge base consisted in a symbolic concepts base coherent with the cortex anatomy model, and in a numeric database representing numeric knowledge about shape or variation for these concepts. The symbolic knowledge base (Fig. 2) was constituted with the most significant entities of frontal, parietal, temporal and occipital regions. The numeric knowledge base represented the shapes of the concepts. It consisted in 3D images for the MRI datasets and of the shape models.

Links between numeric and symbolic elements

A Java application has been implemented in order to highlight the complementarity between the numeric and the symbolic aspects and to provide for browsing of the knowledge base. The first part presented the symbolic knowledge base to the user as a hierarchy based on the is-direct-anatomical-part-of relation. Contextual menus (Fig. 3a) displayed concept properties (e.g., its designations and existence probability) and relations with other concepts (e.g., composition and contiguity with other gyri or sulci). The second part presented the numeric knowledge base, allowing free manipulation of the 3D scenes. The files describing links between symbolic and numeric resources were used by the application to perform synchronization between the symbolic and the numeric parts. It was possible to highlight the numeric elements matching a concept (Fig. 3b); conversely, 
Fig. 2 Some specialization, composition and topological anatomical concepts relationships between
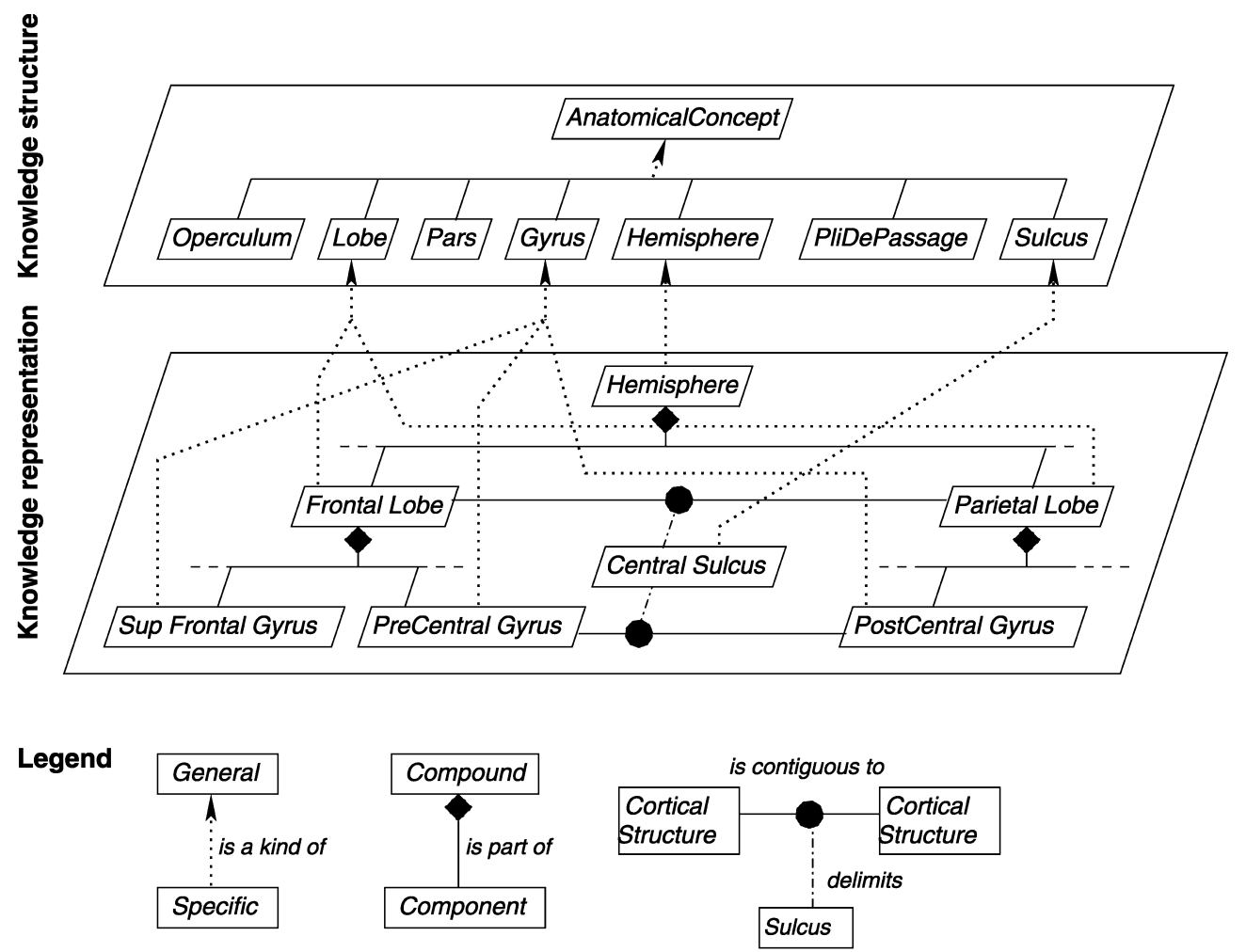

clicking on a numeric element selected the corresponding concept.

\section{Discussion}

The human cerebral cortex has an extraordinarily complex shape and a great deal of attention has been given to its morphological analysis during the last century. The region between two adjacent sides of a cortical fold, called a sulcus, resembles a thin convoluted ribbon embedded in three dimensions. The cerebral sulci have received a great deal of attention primarily because they are believed to be associated with the underlying functional and cytoarchitectonic organization of the cortex $[31,32]$. Furthermore, they are important landmarks that are strongly linked with the boundaries between different cortical regions [10, 25, 30]. A notable example is that of the central sulcus, which is the boundary between its posteriorly located primary somatosensory cortex, belonging to the parietal lobe, and its anteriorly located primary motor cortex, belonging to the frontal lobe. The fact that two-thirds of the cortex is buried within the sulci has complicated the goals of studying the precise geometry of the cortex. One of the most comprehensive cadaver study of the morphology of the major cerebral sulci was reported by Ono et al. [30]. Although this is a comprehensive work with an emphasis on the external view of the sulci, the dissection approach lacked proper demonstration of the inner spatial relationships between different sulci and their 3D structure.
Moreover, no structured symbolic description was proposed.

The information depicted in traditional 2D brain atlases is limited by their ability to convey only static information. Nowhere more than in neuroanatomy is the assimilation of $3 \mathrm{D}$ information often the key to understanding. To date a number of 3D computer-based digital atlases of the brain have been developed [14, 29, 40]. Although many such atlases incorporate sophisticated software tools for viewing and manipulation of images and neuroanatomical structures, only a few of them give symbolic information about the structures $[6$, 27]. We believe that symbolic information and, in particular, symbolic knowledge must parallel image-based spatial information, because it gives meaning to the numeric representation of the cerebral cortex anatomy. This is necessary so that the anatomical knowledge can be automatically used in various contexts such as decision support or data retrieval. We used ontological analysis to clarify the structure of this knowledge. Ontology gathers content theories about the sorts of objects, properties of objects, and relations between objects that are possible in a specified domain of knowledge [8]. In the field of anatomy, it corresponds to the conceptualization of the structures and spaces that constitute the human body, which we extended to the cerebral cortex anatomy following the model proposed by the team of the Digital Anatomist program $[6,35]$ or GALEN [33], and added the numeric aspect. As in other fields, neuroanatomists are compelled to standardize the terminology of their discipline. We used the Neuro- 

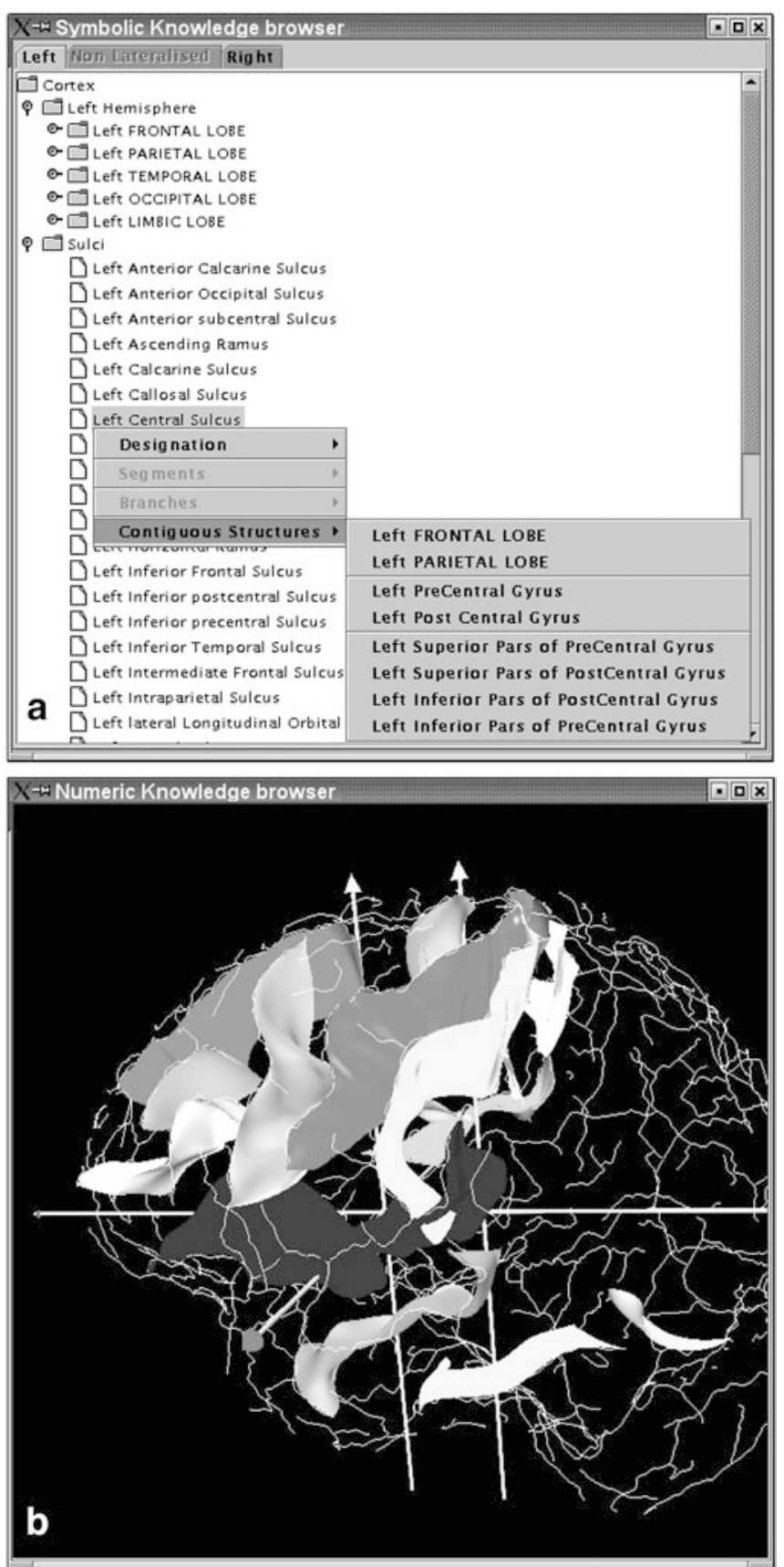

Fig. 3 a Concept properties and relationships in the symbolic browser: example of the left central sulcus. b Three-dimensional numeric scene with the left central sulcus

Names brain hierarchy [5], which is a structured system of neuroanatomical terminology to address deficiencies in the brain terminology presented in Nomina Anatomica [28]. However, we ignored the hierarchy of NeuroNames because of its lack of semantic consistency (specialization and composition relationships are not separated). Finally, the browsing applications proved that XML and VRML are well suited to knowledge representation and sharing.

Increasing amounts of information can be obtained with digital models available from brain atlases, thus facilitating the communication within research laboratories or within healthcare systems and integrated educational programs, through local and global networks $[11,12,13,20,21]$. In the field of image-guided neurosurgery, sulcal information has proven to be very helpful both in supporting the simulation of surgical situations and in the rehearsal of interventions [18, 19]. Using the consistent anatomy of cerebral sulci, the neurosurgeon can achieve access to a subcortical lesion. This so-called transsulcal approach may reduce injury to surrounding normal brain [15]. Thus our model encompasses both the configuration of gyri (for tumor localization for instance) and the configuration of sulci (for access planning for instance). This step only accounts for the shape model of sulci. However, the 3D structures are handled by the system as objects. Therefore, it is also directly compatible with surface representations of the gyri.

However, we feel that teaching or decision-support applications require more formal definition of entities and relationships to establish an explicit distinction between conditions that have to be filled (i.e., identity criteria) and those that are optional and subject to variability. Moreover, the applications may need to know the properties of the symbolic relations we used (specialization, composition and topological). For example, is-anatomical-part-of is transitive because parts of the parts of a compound are parts of the compound, whereas the is-contiguous-to relation is not. But the latter is commutative, and not the former: if A is-contiguous-to $\mathrm{B}$ then B is-contiguous-to A. These additional requirements led us to consider using description logic for conceptual modeling. The complexity of the cerebral cortex topology also required use of the ternary is-separated-from-...-by-relation. This is an original feature since Digital Anatomist and GALEN represent only the delimited structures and not what delimits them $[6,33$, 35]. This formalization step is necessary to allow automatic reasoning (i.e., reasoning that does not require human intervention), and thus use of anatomical knowledge by applications. The formalization we propose overcomes some of the problems encountered by the Digital Anatomist. Particularly, we have seen that representing ternary relationships was needed, as well as representing abstract structures such as the central sulcus and lateralized structures such as the left and right central sulcus by explicitly stating their specificities.

Moreover, our model is designed to be independent from the applications that use it, whereas existing works on the Anatomist project and on the automatic labeling of sulci [24, 34] rely on a specific symbolic model. Therefore, our model could be seen as an external reference usable by the sulci labeling application. Eventually, the application we presented was aiming on the one hand at showing the mapping between the numeric and the symbolic aspects, and on the other at checking that the representation of the knowledge base was usable. In addition, it provides user-friendly browsing of the model by hiding the complexity of the 
text files. It was not an application for visualizing data such as Anatomist [34].

\section{Conclusion}

We have proposed a preliminary study of a symbolic model of brain cortex anatomy. This model provides a better formalization of the relationships between anatomical concepts than the one available in the literature. The symbolic model entities can be associated to numeric entities representing the shape of these structures or their variations among a population of individuals [23]. Our approach is the result of a collaboration between anatomists and computer scientists. With other teams, we are considering the creation of a distributed heterogeneous database. Our model will be used to allow communication between the various sites and to process queries. This database could be useful for performing statistical studies on inter-individual variability. In addition, future developments will address anatomical variability representation by studying different patterns from different subjects and by referencing statistical shape variation models. Furthermore, we will emphasize the usefulness and applicability of this approach in various fields of medicine such as image-guided neurosurgery, research in neuroscience and teaching.

\section{References}

1. Andreasen NC, Cizadlo T, Harris G, et al (1993) Voxel processing techniques for the ante-mortem study of neuroanatomy and neuropathology using magnetic resonance imaging. J Neuropsychiatry Clin Neurosci 5: 121-130

2. Barillot C, Lemoine D, Le Briquer L, Lachmann F, Gibaud B (1993) Data fusion in medical imaging: merging multimodal and multipatient images, identification of structures and 3D display aspects. Eur J Radiol 17: 22-27

3. Bodenreider O, Nelson SJ, Hole WT, Chang HF (1998) Beyond synonymy: exploiting the UMLS semantics in mapping vocabularies. Proc AMIA Symp: 815-819

4. Booch G, Jacobson I, Rumbaugh J, Rumbaugh J (1998) The Unified Modeling Language User Guide. Addison-Wesley, Boston

5. Bowden DM, Martin RF (1995) NeuroNames brain hierarchy. Neuroimage 2: 63-83

6. Brinkley JF, Wong BA, Hinshaw KP, Rosse C (1999) Design of an anatomy information system. IEEE Comp Graph Appl 19: $38-48$

7. Carey R, Bell G (1997) The annotated VRML 2.0 reference manual. Addison-Wesley, Boston

8. Chandrasekaran B, Josephson JR, Benjamins VR (1999) What are ontologies, and why do we need them? IEEE Intell Syst 14: $20-26$

9. Collins L, Evans A (1997) Animal: validation and applications of nonlinear registration-based segmentation. Int J Pattern Rec Artif Intell 8: 1271-1294

10. Duvernoy HM (1991) The human brain: surface, threedimensional sectional anatomy and MRI. Springer, Berlin Heidelberg New York

11. Frasca D, Malezieux R, Mertens P, Neidhardt JPH, Voiglio EJ (1999) Review and evaluation of anatomy sites on the Internet (updated 1999) Surg Radiol Anat 22: 107-110
12. Gibaud B, Garlatti S, Barillot C, Faure E (1998) Computerized brain atlases as decision support systems: a methodological approach. Artif Intell Med 14: 83-100

13. Giffin BF, Drake RL (2000) Gross anatomy of the head and neck and neuroscience in an integrated first-year medical school curriculum. Anat Rec 261: 89-93

14. Greitz T, Bohm C, Holte S, Erricson L (1991) A computerized brain atlas: construction, anatomical content and some applications. J Comput Assist Tomogr 15: 26-38

15. Harkey HL, Al-Mefty O, Haines DE, Smith RR (1989) The surgical anatomy of the cerebral sulci. Neurosurgery 24: 651-654

16. Harold ER, Means WS (2002) XML in a nutshell, 2nd edn. O'Reilly, Cambridge, Mass

17. Höhne KH, Pflesser B, Pommert A, et al (1995) A new representation of knowledge concerning anatomy and function. Nat Med 6: 506-511

18. Jannin P, Morandi X, Fleig OJ, et al (2002) Integration of sulcal and functional data for multimodal neuronavigation. J Neurosurg 96: 713-723

19. Jannin P, Seigneuret E, Morandi X, et al (2000) Repérage sulcal et neuronavigation dans la chirurgie des cavernomes supratentoriels. Neurochirurgie 46: 534-540

20. Kikinis R, Shenton ME, Iosifescu DV, et al (1996) A digital brain atlas for surgical planning, model-driven segmentation, and teaching. IEEE Trans Vis Comput Graph 2: 232-241

21. Kikinis R, Gleason PL, Moriarty TM, et al (1996) Computerassisted interactive three-dimensional planning for neurosurgical procedures. Neurosurgery 38: 640-649

22. Le Goualher G, Barillot C, Bizais Y (1997) Modeling cortical sulci with active ribbons. Int $\mathbf{J}$ Pattern Rec Artif Intell 11: $1295-1315$

23. Le Goualher G, Procyk E, Collins DL, et al (1999) Automated extraction and variability analysis of sulcal anatomy. IEEE Trans Med Imag 18: 206-217

24. Mangin JF, Régis J, Poline JB, et al (1998) Place de l'anatomie dans la cartographie fonctionnelle du cerveau. Ann Inst Pasteur Actual 9: 243-258

25. Naidich TP, Valavanis AG, Kubik S (1995) Anatomic relationships along the low-middle convexity. I. Normal specimens and magnetic resonance imaging. Neurosurgery 36: 517-532

26. Narayan S, Sensharma D, Santori EM, Lee AA, Sabherwal A, Toga AW (1993) Animated visualization of a high resolution color three dimensional digital computer model of the whole brain. Int J Biomed Comput 32: 7-17

27. Natarajan K, Claridge E, Davis DN (1996) The symbolic atlas of the brain: handling non-visual information associated with neuroanatomy. Med Inform 21: 1-22

28. Anon (1989) Nomina anatomica, 6th edn. Churchill-Livingstone, Edinburgh

29. Nowinski WL, Fang A, Nguyen BT, et al (1997) Multiple brain atlas database and atlas-based neuroimaging system. Comput Aided Surg 2: 42-66

30. Ono M, Kubik S, Abernathey C (1990) Atlas of the cerebral sulci. Thieme, New York

31. Penfield W, Boldrey E (1937) Somatic motor and sensory representation in the cerebral cortex of man as studied by electrical stimulation. Brain 60: 389-444

32. Rademacher J, Caviness VS, Steinmetz H, Galaburda AM (1993) Topographical variation of the human primary cortices: implications for neuroimaging, brain mapping, and neurobiology. Cereb Cortex 3: 313-319

33. Rector AL, Nowlan WA, and the GALEN project (1994) The GALEN project. Comput Meth Prog Biomed 45: 75-78

34. Rivière D, Mangin JF, Papadopoulos-Orfanos D, Martinez JM, Frouin V, Régis J (2002) Automatic recognition of cortical sulci of the human brain using a congregation of neural networks. Med Image Anal 6: 77-92

35. Rosse C, Mejino JL, Modayur BR, Jakobovits R, Hinshaw KP, Brinkley JF (1998) Motivation and organizational principles for anatomical knowledge representation: the Digital Anatomist symbolic knowledge base. J Am Med Inform Assoc 5: $17-40$ 
36. Sastre-Janer FA, Regis J, Belin P, et al (1998) Three-dimensional reconstruction of the human central sulcus reveals a morphological correlate of the hand area. Cereb Cortex 8: 641647

37. Schaltenbrand G, Wahren W (1977) Atlas for stereotaxy of the human brain. Thieme, Stuttgart

38. Talairach J, Tournoux P (1996) Co-planar stereotactic atlas of the human brain. Thieme, Stuttgart

39. Thompson PM, Schwartz C, Lin RT, Khan AA, Toga AW (1996) Three-dimensional statistical analysis of sulcal variability in the human brain. J Neurosci 16: 4261-4274
40. Tiede U, Bomans M, Hohne KH, et al (1993) A computerised 3-dimensional atlas of the human skull and brain. AJNR Am J Neuroradiol 14: 551-559

41. Zilles K, Schleicher A, Langemann C, et al (1997) Quantitative analysis of sulci in the human cerebral cortex: development, regional heterogeneity, gender difference, asymmetry, intersubject variability and cortical architecture. Hum Brain Mapping 5: 218-221 